\title{
Out-of-Plane Strain Effects on Physically Flexible FinFET CMOS
}

\author{
Mohamed T. Ghoneim, Student Member, IEEE, Nasir Alfaraj, Student Member, IEEE, Galo A. Torres-Sevilla, \\ Student Member, IEEE, Hossain M. Fahad, Member, IEEE, and Muhammad M. Hussain, Senior Member, IEEE
}

\begin{abstract}
We present a comprehensive electrical performance assessment of hafnium silicate $\left(\mathrm{HfSiO}_{\mathrm{x}}\right)$ high- $\mathrm{K}$ dielectric and titanium nitride (TiN) metal gate integrated FinFET-based complementary-metal-oxide semiconductor (CMOS) on flexible silicon-on-insulator (SOI). The devices were fabricated using state-of-the-art CMOS technology and then transformed into flexible form by using a CMOS-compatible mask-less deep reactive-ion etching (DRIE) technique. Mechanical out-of-plane stresses (compressive and tensile) were applied along and across the transistor channel lengths through a bending range of 0.5 to 5 cm radii for n-type and p-type FinFETs. Electrical measurements were carried out before and after bending, and all bending measurements were taken in the actual flexed (bent) state to avoid relaxation and stress recovery. Global stress from substrate bending affects the devices in different ways compared to the well-studied uniaxial/biaxial localized strain. The global stress is dependent on the type of channel charge carriers, the orientation of the bending axis, and the physical gate length of the device. We therefore outline useful insights on the design strategies of flexible FinFETs in future free-form electronic applications.
\end{abstract}

\section{Index Terms - Flexible, CMOS, FinFET, strain.}

\section{INTRODUCTION}

THE Internet of Everything (IoE) envisions the connection of living beings (e.g. humans, plants, animals, and birds) and objects to the cloud, where vital signs, activity rates, and other aspects of daily life can be monitored and analyzed to inform decisions and improve lifestyles. In that sense, IoE devices require close proximity to the human body, fast decision making capability, and low heat dissipation. They must have low power consumption (dynamic and static), high performance, and flexibility. Subthreshold swing (SS) is a critical parameter to this end because it affects the switching speed of a transistor and its dynamic power consumption [1].

The nonplanar 3D architecture of a FinFET device alse enables low-power operation and further scaling, to cope with Moore's law [2, 3]. FinFET devices are already commercially available at higher technology nodes (22 and $14 \mathrm{~nm}$ ). The international technology roadmap for semiconductors (ITRS) predicts a physical gate length requirement of $7 \mathrm{~nm}$ by 2025 [4]. Although such state-of-the-art CMOS electronics exhibit

This publication is based upon work supported by the King Abdullah University of Science and Technology (KAUST) Office of Sponsored Research (OSR) under Award No. CRG-1-2012-HUS-008. We acknowledge Seneca J. Velling for proof reading this manuscript. All authors are with the Integrated Nanotechnology $\mathrm{Lab}$ and Integrated Disruptive Electronic Applications (IDEA) Lab, Electrical Engineering, Computer Electrical Mathematical Science and Engineering Division, King Abdullah University of Science and Technology, Thuwal 23955-6900, Saudi Arabia (e-mail: muhammadmustafa.hussain@kaust.edu.sa). unparalleled advantages, they are rigid and bulky making them unsuitable for IoE applications. CMOS electronics thus need to be of freeform - physically flexible and stretchable.

In this work, we applied various mechanical stresses through bending to the flexed FinFET devices to assess their suitability for flexible electronics applications, including IoE and wearable systems, where flexibility and high performance are essential features for components. We quantify and analyze the effect of out-of-plane stress on key performance metrics to provide insights into the integration feasibility of state-of-the-art CMOS devices with new emerging applications.

\section{EXPERIMENTAL}

We used SOI substrates with a $90 \mathrm{~nm}$ SOI layer (100) and a gate first approach. A summary of the main fabrication steps is depicted with a cross-section showing fin internal structure in Fig. 1(a-c), where the inset showing fins orientation. Fig. 1(df) show the main flexing steps to transform the rigid substrate into a 50- $\mu \mathrm{m}$-thick flexible form. We used a Keithley 4200SCS Semiconductor Characterization System on a manual SemiProbe probe station, as well as curved aluminum surfaces for device characterization (Fig. 1(g)). Bending was done along the fins (i.e., where the bending axis is parallel to the line connecting source and drain) and across the fins (i.e., where the bending axis is perpendicular to the fin length).

\section{RESULTS AND DISCUSSION}

\section{A. Silicon's Bending Ability and Limitations}

Fracture strength determines the overall mechanical stability of a flexible system. The three-point bending test is widely used to assess the fracture strength of a substrate. For silicon thicknesses below $100 \mu \mathrm{m}$, the linear elastic bending beam theory cannot provide an accurate estimation of fracture strength because thin substrates produce a nonlinear deflection-load relationship that is used to estimate fracture strength [8].

In 2015, Liu et al. introduced the large deflection theory of beam to account for this nonlinearity [9]. They offered important insights for theoretical limitations of flexible silicon thinner than $100 \mu \mathrm{m}$. Furthermore, based on the application's required bending radius, the thickness of the flexible silicon substrate must be adjusted such that the applied stress is determined as

$$
\sigma=Y \varepsilon(1)
$$

Where $\sigma$ is the stress in Pascals, $Y$ is Young's modulus, and $\varepsilon$ is the nominal strain, which can be calculated as 


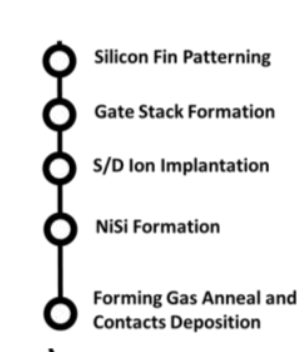

a)

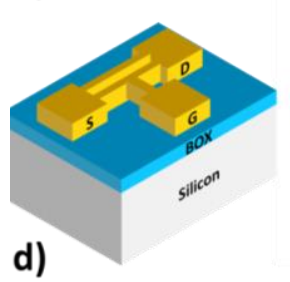

b)
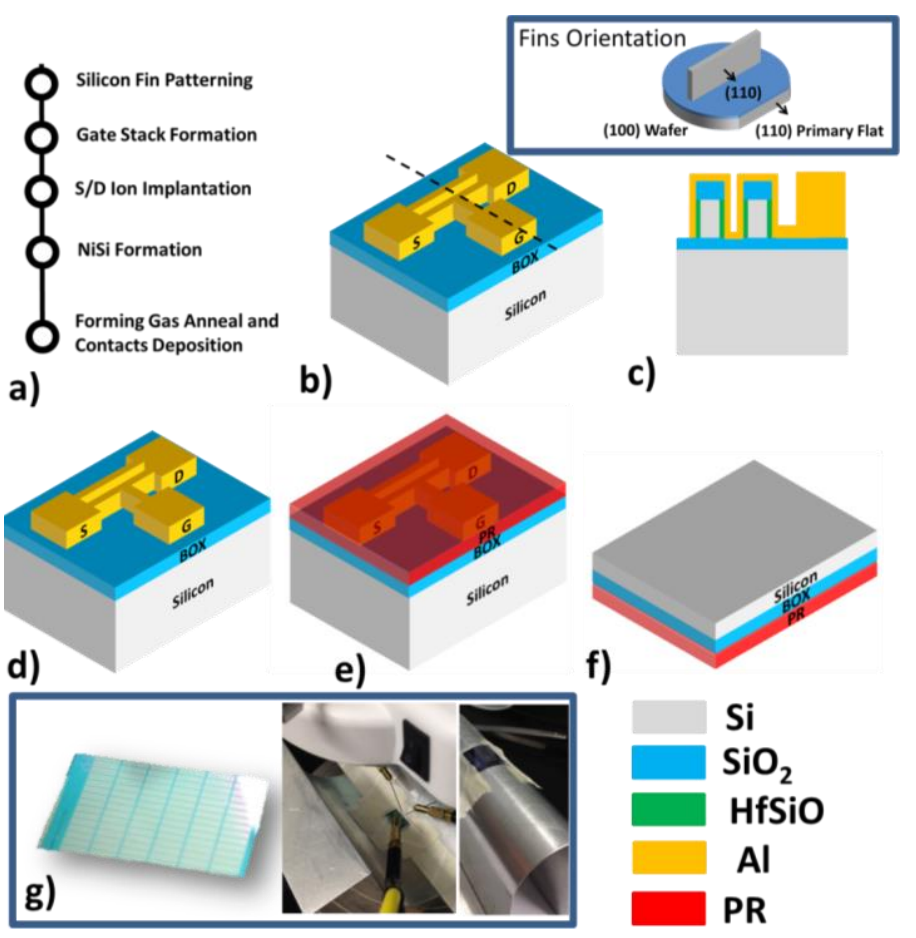

Fig. 1. Fabrication flow (a-f) and characterization setup (g) of flexible FinFET.

$$
\varepsilon_{\text {nominal }}=\frac{t}{2 R}
$$

Where $t$ is the substrate thickness and $R$ is the bending radius.

The applied stress needs to be lower than the fracture stress for mechanical integrity. Reference [9] showed that for a 50$\mu \mathrm{m}$-thick silicon fabric, the fracture stress is about $1.1 \mathrm{GPa}$. Substituting a (100) silicon Young's modulus of $128 \mathrm{GPa}$ [10] in Equation (1), and the resulting strain value from Equation (2), we obtain a minimum bending radius for the $50-\mu \mathrm{m}$-thick flexible silicon fabric of approximately $3 \mathrm{~mm}$, which decreases with a decreasing thickness. Therefore, a plain flexible silicon substrate that is 50 - $\mu$ m-thick or thinner with a bending radius of more than $3 \mathrm{~mm}$ will safely operate below the fracture stress level. We emphasize that these results are for a bare silicon substrate with no additive layers or patterns for devices. Therefore, based on material properties, substrate thickness, and the bending radius necessary for a specific application, we can determine the most suitable approach and material system for fabricating a thin substrate. These results identify important boundary limitations and guarantee that a bending of $0.5 \mathrm{~cm}$ is reasonably far from fracture stress regime. In most cases where flexible electronics are required to wrap around curvilinear objects, such as covering a fingertip or an arm, a bending radius of $3 \mathrm{~mm}$ would suffice. Moreover, continuing to reduce the substrate's thickness is another option that would enable us to decrease the bending radii and achieve a similar nominal strain corresponding to stress values below the fracture limit. Nonetheless, polymeric electronics exhibit better flexibility and might be a more feasible option for extreme applications that require folding of the substrate. Sekitani et al. demonstrated ultra-flexibility using polymeric substrates and Pentacene semiconductor- based organic-thin-film transistors (OTFT) that can bend at $100 \mu \mathrm{m}$ radius for various applications [11].

\section{B. Effect of Bending Axis Orientation, Stress Type, Carrier Type on FinFET Performance}

Empirical analysis of the effects of bending direction and bending axis orientation on the dimensions of the fins, representing the carrier channels, should provide useful insights into the feasibility of the integration of state-of-the-art CMOS devices into the aforementioned IoE applications. Silicon's elastic modulus (128 GPa for (100) silicon) allows us to relate the (100) silicon Poisson's ratio of 0.17 [12] to the change in dimension through the following basic equation

$$
v \approx \frac{\Delta L^{\prime}}{\Delta L}
$$

Where $\Delta L^{\prime}$ is transverse strain and $\Delta L$ is the axial strain, as shown in Fig. 2(a). Fig. 2(b) shows the finite element method (FEM) simulation results for surface tensile and compressive stresses, anticipating that compressive stress has a greater impact on device behavior (nearly doubling applied stress at the surface containing the devices), while illustrating the two bending conditions (across and along the channel). Fig. 3 shows the effect of compressive and tensile stresses on the saturation transfer $\mathrm{I}-\mathrm{V}$ characteristics of a sub-micron physical gate length $\left(L_{g}\right)$ n-type and p-type FinFETs bent at \pm 3 $\mathrm{cm}$ along the channel (Fig. 3(a-b)), and FinFETs bent at \pm 5 $\mathrm{cm}$ across the channel (Fig. 3(c-d)), corresponding to $0.08 \%$ and $0.05 \%$ nominal strain, respectively. The measurements confirm that compressive stress always has a more pronounced effect for both devices-for different channel carriers types and for both bending axes (i.e., along and across the channel). We note the change of bending dimensions analysis by the output $\mathrm{I}-\mathrm{V}$ characteristics curves, which showcase the pattern on a linear scale (Fig. 3(e-f)). Based on changes in the fin/channel dimensions depicted in Fig. 2(a), the pattern is expected. However, this pattern was not meticulously followed in the case of along-the-channel bending, indicating that the variation is better explained as an anomaly in behavior within a specific variation range than a consistent trend. Fig. 4(a-c) show the current flowing between the three terminals (Source/Drain/Gate) in the FinFET depicted in Fig. 3 (c) on a linear scale. In unbent and tensile stress cases, as the electric field in the channel becomes stronger, the gate leakage is almost constant and current flows between source and drain prominently. On the other hand, in case of compressive stress, the magnitude of both subthreshold leakage in the channel and gate leakage increase, and as the electric field between the gate and the channel increases, the gate leakage current linearly increases. This effect is better expressed in the subthreshold regime due to lower current levels between the source and drain, where gate leakage is of comparable magnitude. In all cases, the vector sum of currents is zero, except where the individual components are flowing, indicative of a compromised gate dielectric integrity under compressive stressing. For instance, Fig. 4(d-f) show that for unbent and tensile stress, the off current (subthreshold channel current and gate leakage) is due to current flowing from Drain 
$\left(\mathrm{V}_{\mathrm{DD}}\right)$ into Source (ground) and Gate (ground) terminals, remaining nearly constant as Gate voltage increases until switching occurs. On the other hand, under compressive stress, $\mathrm{I}_{\mathrm{OFF}}$ is initially higher and gate leakage continues to increase linearly with increasing Gate voltage, indicating conduction through the gate dielectric as current now flows from Gate and Drain into the Source in subthreshold regime and continues even after the transistor is switched ON. This confirms that the effect results from a compromised dielectric integrity caused by compressive stress.

Localized strain enhancement in CMOS transistors has been extensively studied for decades [13], with the assumption that different carriers will respond to stress differently. Wesler et al. used energy band diagrams to explain the different responses to stress with respect to carrier types [14]. For instance, in the suggested $\mathrm{SiO}_{2} / \mathrm{Si} / \mathrm{Si}_{0.7} \mathrm{Ge}_{0.3}$ strained structure, a barrier hole well in the valence band corresponds to a surface electron well in the conduction band. The strained silicon surface energy band structure is similar to the bent FinFETs case, where the stress gradient along the silicon thickness due to bending induces a strain effect, rather than the SiGe layer, influencing the effective masses $\left(m^{*}\right)$ of charge carriers due to the deformations in the constrained/stretched energy $(E)$ bands $\left(m^{*} \alpha\left(\frac{d^{2} E}{d K^{2}}\right)^{-1}\right.$, where $K$ is proportional to crystal momentum). However, due to band bending in different directions, tensile and compressive strains should form different barrier and surface well structures, depending on carrier type. This affects the transport properties especially at the edges of the channel (or fins) where lateral stress values are relatively higher and extra surface and barrier wells for carriers are likely to form. Oppositely charged free carriers behave differently depending on the bending axis (Fig. 3). Further FEM analysis, as represented by Fig. 5(a), shows a two-dimensional stress distribution in a fin bent across the channel and along the channel. Devices with larger dimensions would show similar stress distribution. The critical point would be for further scaled-down devices where the higher stress regions merge to form different stress patterns, which might stress the whole channel and lead to different behavior. Comparing the two stress profiles, it is clear that bending across the channel leads to different stress distribution than bending along the channel. As can be deduced from the results shown in Fig. 3, electrons are more sensitive to stress when the channel is bent along its length, while holes are more sensitive when the bending axis is perpendicular to the channel length. Because CMOS-based technology utilizes both n- and p-type field-effect transistors (FETs) on the same wafer, the assessed degradation should be the worst-case scenario for both of the two channel types. Furthermore, localized stress due to SiGe source and drain has historically been used to strain the channel and enhance mobility. On the other hand, a dissimilar effect takes place in this study. This is because empirical stress imposed by out-of-plane bending causes a different stress distribution profile and different magnitudes compared to localized stress when both are exposed to same strain values (Fig. 5(b)). The inset in Fig. 5(b) shows the calculation of the displacement required for specific strain and the resulting stress profiles for out-of-plane and in-plane fins, stressed at the same strain values. Using equations (1-2), the stress regime for 0.5 to $5 \mathrm{~cm}$ bending radii is 80 to $800 \mathrm{MPa}$, corresponding to an intermediate stress range relatively lower than that used in strained silicon to engineer mobility enhancements [15]. Still there were cases of mobility enhancements for long-channel devices flexed along the channel, but due to the complexity of the testing setup and sources of variation while contacting the devices, the conclusion would be an anomaly within $\pm 5 \%$ for shortchannel devices. Other key switching properties are summarized in Table I for short-channel FinFETs bent at \pm 5 $\mathrm{cm}$ bending radius.

Table I

Key switching properties of short-channel FinFETs under out-ofplane stress due to $\pm 5 \mathrm{~cm}$ bending radius

$\begin{array}{ccc}\text { Parameter } & \text { Method } & \begin{array}{c}\% \\ \text { change }\end{array} \\ \text { SS } & \min \left(\Delta V_{G S} / \log \left(I_{D S 1} / I_{D S 2}\right)\right) & \sim 10 \% \\ \mathbf{V}_{\mathbf{T H}} & I_{D S} / g_{m}-V_{G S} \mid @ \max g_{m} & \sim 10 \% \\ \text { DIBL } & \left(V_{T H, s a t}-V_{T H, l i n}\right) /\left(V_{D D}-V_{D S, l o w}\right) & \pm 50 \% \\ \boldsymbol{g}_{\text {m,max }} & \partial I_{D S} / \partial V_{G S} & -40 \% \\ \mathbf{I}_{\mathbf{O N}} / \mathbf{I}_{\text {OFF }} \text { Ratio } & I_{O N} @ V_{D S}=V_{G S}=V_{D D} & \sim 33 \% \\ & I_{O F F} @ V_{D S}=V_{D D}, V_{G S}=0 \mathrm{~V} & \end{array}$

The large variation in the drain-induced barrier lowering (DIBL) implies that the devices suffered from significant threshold voltage $\left(V_{T H}\right)$ shifts when functioning in the saturation regime. An increment under compressive stress implies that the device suffers higher leakage currents due to a relatively larger reduction in $V_{T H}$. A decrease in DIBL under tensile stress would lead to a device with relatively larger $V_{T H}$ than designed, and, consequently, lower overdrive voltage ( $V_{D D}-V_{T H}$, where $V_{D D}$ is the supply voltage) and on-state currents $\left(I_{O N}\right)$. The decrease in the peak transconductance $\left(g_{m}\right)$ would degrade the performance of a transistor device while operating as an amplifier, because the gain of an amplifier is proportional to the transconductance of the device. Ideally, ICs are designed to tolerate $10 \%$ deviation in circuit parameters. Therefore, for anomalies greater than $10 \%$, serious considerations are essential when integrating such high-performance devices in flexible ICs that are intended to function properly under various bending conditions. This can be mitigated by redundant compensation elements to adjust the parameters. Current design for yield in deep-submicron technologies already uses similar techniques to comply with $3 \sigma$ and $6 \sigma$ standards ( $\sigma$ measures variability), accommodating process variations for extremely scaled emerging nodes. For instance, adding a backup transistor connected in parallel when needed to enhance the circuit current as the original transistor experiences increased $V_{T H}$ and lower current. The observed trend for increased gate leakage with bending can be utilized to sense the leakage current and determine the corresponding parameter deviation, allowing us to make dynamic decisions to engage added components and correct 
the deviation effect.

To extract the effective mobility $\left(\mu_{e f f}\right)$, we use:

$$
\mu_{e f f}=\frac{g_{d} L_{g}}{W Q_{n}}(4)
$$

Where $g_{d}$ is the drain conductance, $Q_{n}$ is the mobile charge density in the channel, and $W$ is the channel width.
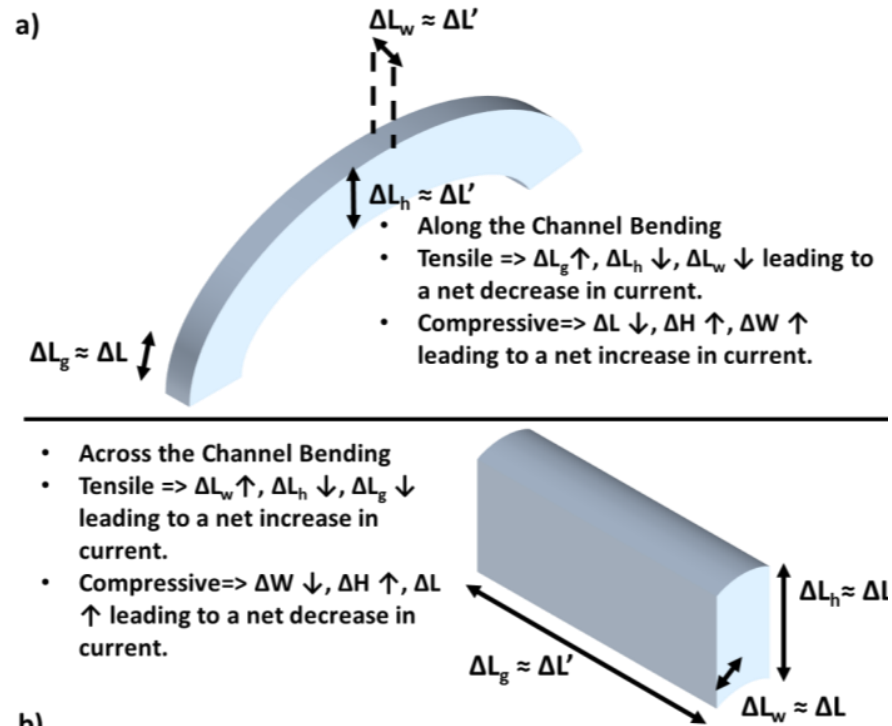

b)

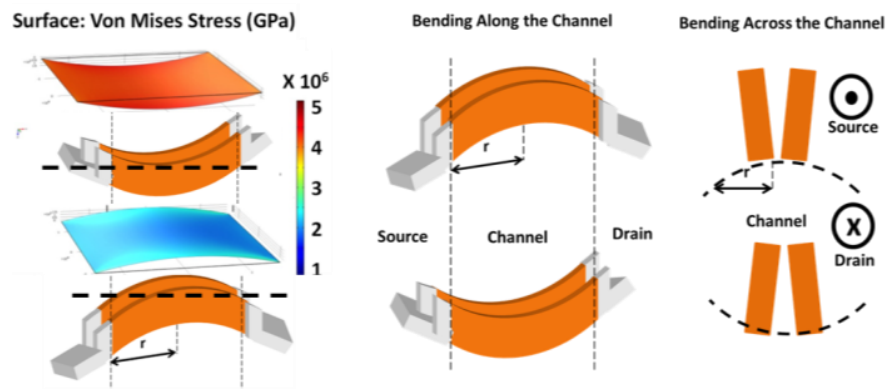

Fig. 2. Illustrations on changes in fin dimensions (a), and FEM simulations for Von Mises stress distribution (b).

Fig. 6 depicts the extracted effective mobility values versus $\mathrm{L}_{\mathrm{G}}$ for tensile and compressive bent $n$ - and p-type FinFETs. Conclusively, FinFETs with longer $L_{G}$ exhibited higher deviations from the unbent benchmark, for both majority carrier and applied stress types. For instance, a representative n-type FinFET showed a deviation of $13 \%$ for an $L_{G}$ of $10 \mu \mathrm{m}$, compared to a $2.3 \%$ deviation for $L_{G}$ of $110 \mathrm{~nm}$. Similarly, a representative p-type FinFET had a $90 \%$ deviation for $\mathrm{L}_{\mathrm{G}}$ of $10 \mu \mathrm{m}$ and $2.7 \%$ deviation for $L_{G}$ of $250 \mathrm{~nm}$. Although the long-channel p-type FinFET exhibited a $90 \%$ deviation in its effective mobility, their functionality is still attested as a switch, as evidenced in Fig. 6(c). Under both applied stress types, the on-to-off current ratio $\left(I_{O N} / I_{O F F}\right)$ was about $10^{3}$ with low gate leakage.

In ultra-large scale integration (ULSI), the flowing drain current in the on-state $\left(I_{O N}\right)$ and the off-state $\left(I_{O F F}\right)$ are critical properties of every device. Depending on the magnitudes of $I_{O N}, V_{D D}$, and gate capacitance $\left(C_{G} \approx\right.$ oxide capacitance $\left.\left(C_{O X}\right)\right)$, the intrinsic delay $\left(t_{d}\right)$ of the device is determined as $t_{d}=$ $C V_{D D} / I_{O N}$. Fig. 7(a) shows the variation of the gate delay with the $\mathrm{I}_{\mathrm{ON}} / \mathrm{I}_{\mathrm{OFF}}$ ratio for a representative p-type FinFET device. The higher the $I_{O N}$, the faster the circuit and the total capacitance of the circuit, including parasitic, is proportional to the time constant $(\tau)$, which affects the speed at which the circuit can operate.

The ultimate goal of strain engineering is to increase $\mathrm{I}_{\mathrm{ON}}$ through mobility enhancements. A FET current is given by:
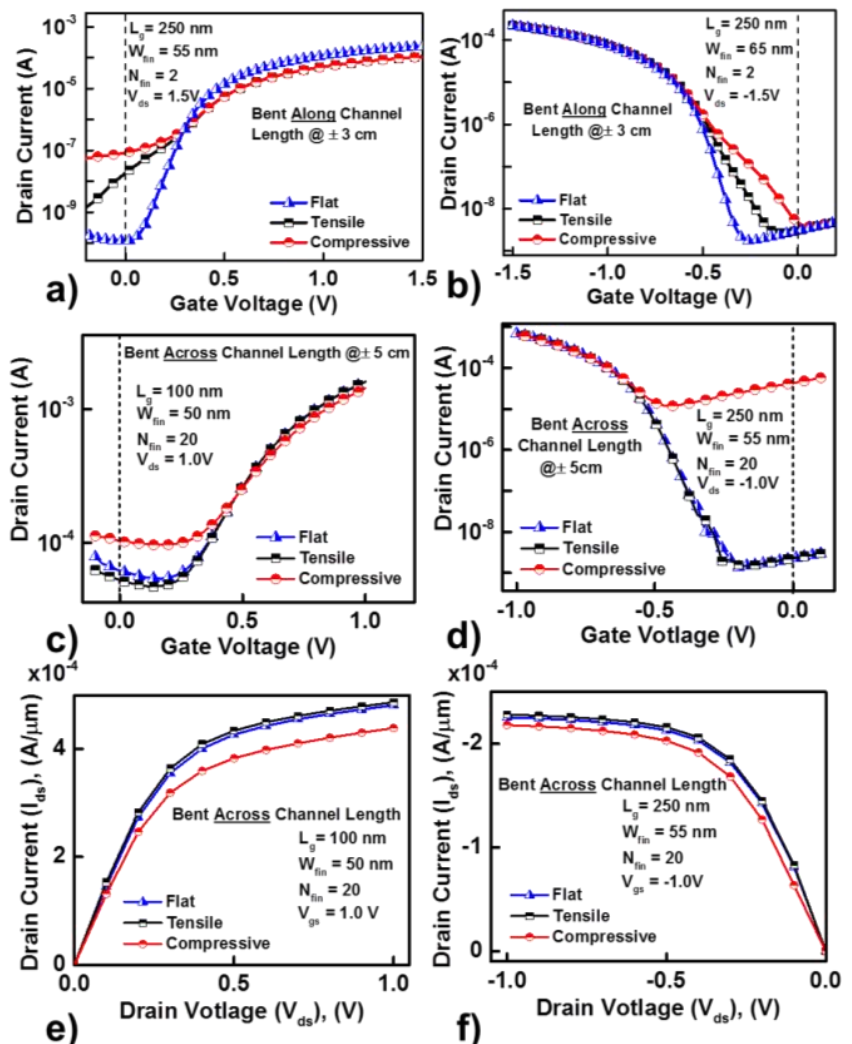

f)

Fig. 3. Transfer (a-d) and output (e-f) plots of FinFETs of various dimensions under varied stress conditions and bending axes.

$$
I_{D S}=\frac{W C_{O X} \mu_{e f f}}{L_{G}}\left[V_{D S}\left(V_{G S}-V_{T H}\right)-\frac{V_{D S}^{2}}{2}\right]
$$

$I_{O N}$ corresponds to the saturation current $\left(\mathrm{I}_{\mathrm{SAT}}\right)$ when $\mathrm{V}_{\mathrm{DS}} \geq$ $V_{G S}-V_{T H}$ and is approximately (discarding short channel modulation effect) given as

$$
I_{O N}=\frac{W C_{O X} \mu_{e f f}}{2 L_{G}}\left(V_{G S}-V_{T H}\right)^{2}(6)
$$

For a representative short-channel p-type FinFET $\left(L_{G}<250\right.$ $\mathrm{nm})$, the change in $I_{O N}$ is less than $1 \%$ under both tensile $(1.5$ $\mathrm{cm}$ bending radius) and compressive stress $(-3 \mathrm{~cm}$ bending radius). This is justified by the insignificant deviations in $\mu_{e f f}$ and $V_{T H}$ while other parameters are physical.

Even for a long-channel p-type FinFET device $\left(L_{G}=10\right.$ $\mu \mathrm{m})$ - where the change in calculated $\mu_{\text {eff }}$ is was about $90 \%$ - the changes in $I_{S A T}$ were $-2.4 \%$ for tensile stress and $1.5 \%$ for compressive stress (both at $2.25 \mathrm{~cm}$ bending radius), at $V_{D S}=V_{G S}=-1.5 \mathrm{~V}$. The extreme variation in $\mu_{\text {eff }}$ did not translate into current variation-although the change in $V_{T H}$ while operating in the saturation regime (extracted using the Ghibaudo method for short-channel devices, i.e. the intercept 
with $V_{G S}$ axis from $I_{D S} / \sqrt{ } g_{m, s a t} v s$. $V_{G S}$ plot [16]) is less than $8 \%$ in worst-case compressive stress, and less than $1 \%$ for tensile stress (at $2.25 \mathrm{~cm}$ bending radii).

Hence the calculated mobilities do not reflect actual degradation, which is attributed to the susceptibility of the measurement used for mobility calculations to noise, namely the strong dependence of mobility on $g_{d}$ which is measured at very low drain voltages (between 25 and $50 \mathrm{mV}$ ).
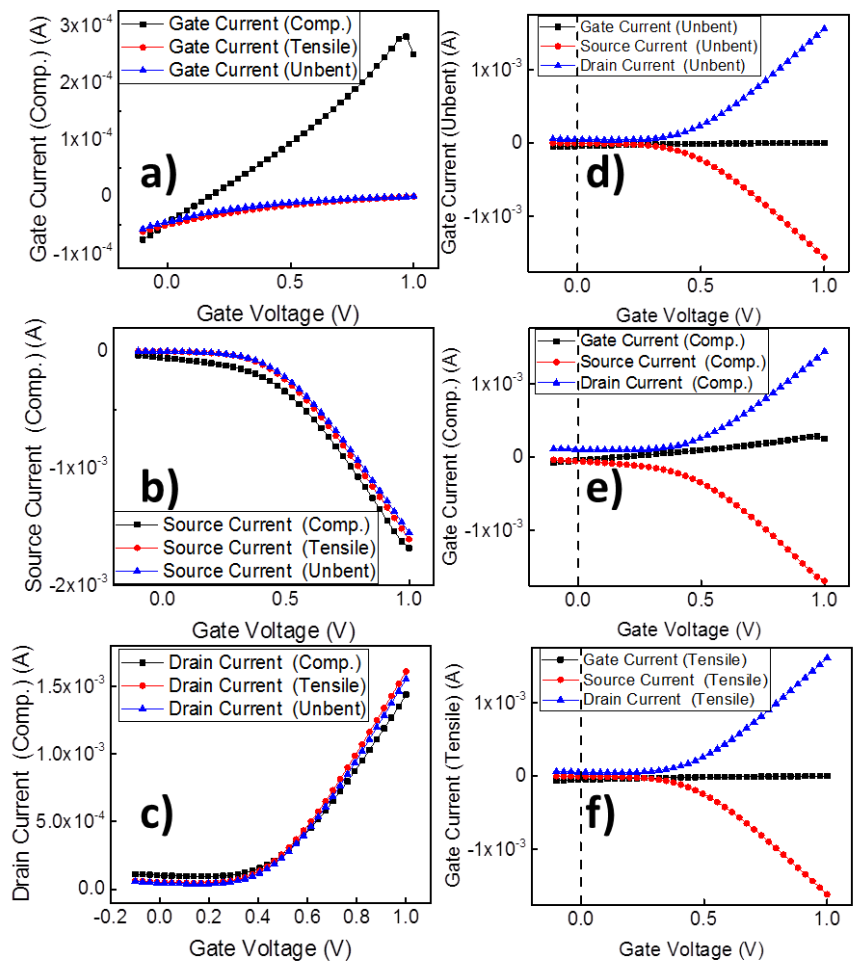

Fig. 4. Closer analysis of FinFET in Fig. 3(c) when bent at $\pm 5 \mathrm{~cm}$ radii, showing (a-c) the variation in currents through all terminals, and (d-f) the current flows through the terminals of the device.

Physical scaling compromises the reliability and physical limits of the gate oxide in terms of interface quality and defect density. Therefore, high- $\kappa$ dielectrics have been used to replace $\mathrm{SiO}_{2}$. The FinFETs in this work utilize few nanometers of $\mathrm{HfSiO}_{\mathrm{x}}$ high- $\kappa$ dielectric. A consistent trend of increasing gate leakage between the gate terminal and the source of a FinFET has been observed, especially under tensile stress for a representative short-channel $250 \mathrm{~nm}$ p-type FinFET (Figs. 7(b-c)). This is in line with previous observations by Choi et al. on the reduction in the work function of TiN due to tensile out-of-plane bending which reduces the Gate/Oxide barrier height causing higher leakage currents [17].

This does not affect the functionality of the device due to the orders of magnitudes difference between $\mathrm{I}_{\mathrm{DS}} v s$. $\mathrm{V}_{\mathrm{DS}}$, as seen in Fig. 7(d). Nonetheless, we found that FinFET devices with various dimensions had still been functioning after long bending durations (about one year), as depicted in Fig. 8. The presence of leakage currents is a major issue in deepsubmicron technologies. $I_{O F F}$ is highly dependent on $V_{T H}$, dielectric integrity, and operation temperature. Device operation dependency on temperature was not studied in this work. $\mathrm{I}_{\mathrm{OFF}}$ is inversely proportional to an exponential function in $V_{T H}\left(I_{O F F} \alpha e^{\left(V_{G S}-V_{T H}\right)}\right)$. Therefore, leakage current is strongly affected by shifts in $V_{T H}$ and might require circuit correction (although the variation in our case is within 10\%). The degradation in dielectric integrity is evident from the increasing gate leakage with increasing bending radius. Tensile stress increased leakage significantly at $0.5 \mathrm{~cm}$ bending radius along the channel.

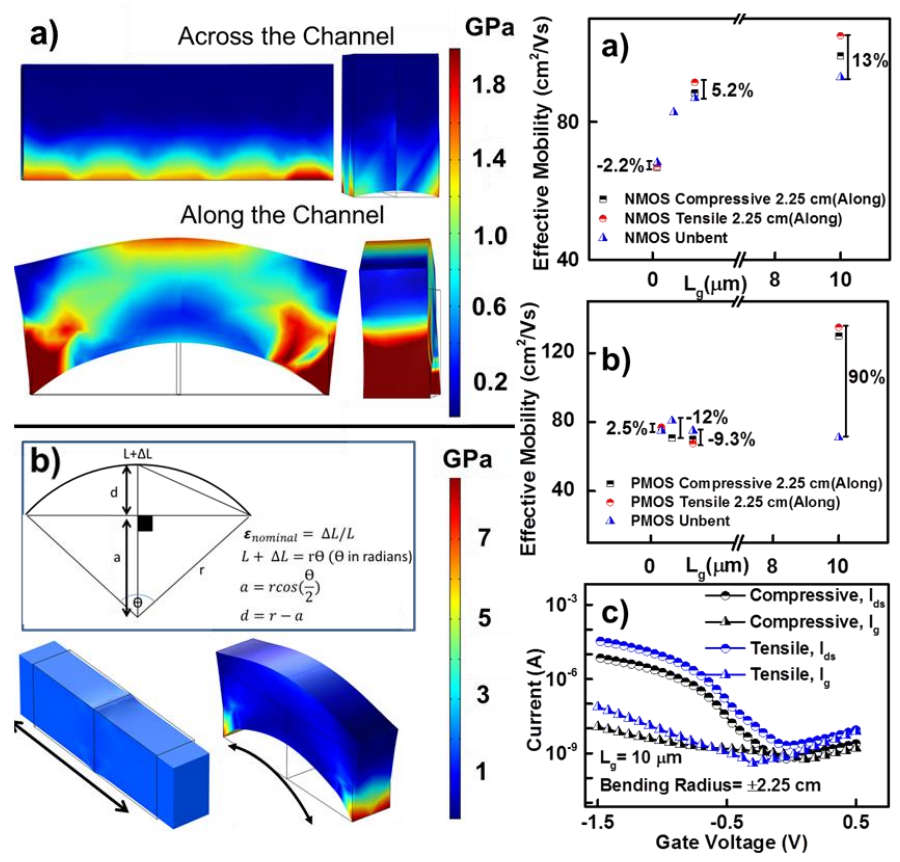

Fig. 5. FEM stress profile for along and across the channel bending (a) and in-plane/out-of- plane stress (b).

Fig. 6. Mobility $v s$. gate length (a-b) and functional device with $90 \%$ mobility change (c).

This means that in a battery-operated IoE system, where the device is in standby mode most of the time and leakage power dominates, the useful time before a re-charge would significantly shortened. Furthermore, the effect is not direct due to the existing positive feedback loop between flowing current and temperature. The higher currents increase the temperature. The relation between the temperature and the semiconductor band gap is given by the Varshni's equation:

$$
E_{g}(T)=E_{g}(0)-\frac{T^{2}}{T-\beta}
$$

Where $E_{g}$ is the band gap in $\mathrm{eV}, T$ is the temperature, and $\beta$ is a material property. This in turn reduces the $V_{T H}$, and consequently, increases the current further. This feedback loop is a common cause of thermal runaway failures in ICs. Therefore, the increment in $I_{O F F}$ can yield more sever effects and requires closer circuit level studies to prevent the potential for eventual failures during the useful lifetime of a system.

At the device level, still no observable degradation in switching behavior under various bending conditions is present. The gate leakage is $1000 \times$ less than the drain currents, i.e. a minute increase in leakage will not significantly affect $I_{O N}$, preserving functionality.

\section{Residual Stress vs. Effective Stress}

In microcrystalline silicon transistors, Dong et al. showed 
the effect of bending of flexible TFTs [19]. All measurements were taken in the flat position after the sample had been exposed to various bending stress types and various durations. We anticipate this work to be an important milestone in assessing out-of-plane stress effects on transistor characteristics. However, the study was focused on residual stress rather than real-time actual stress measurements.

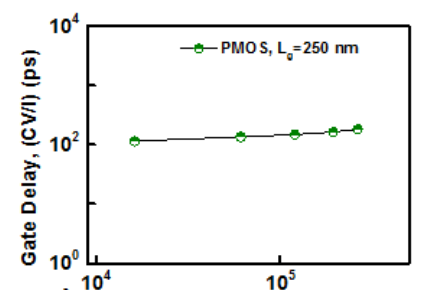

a)
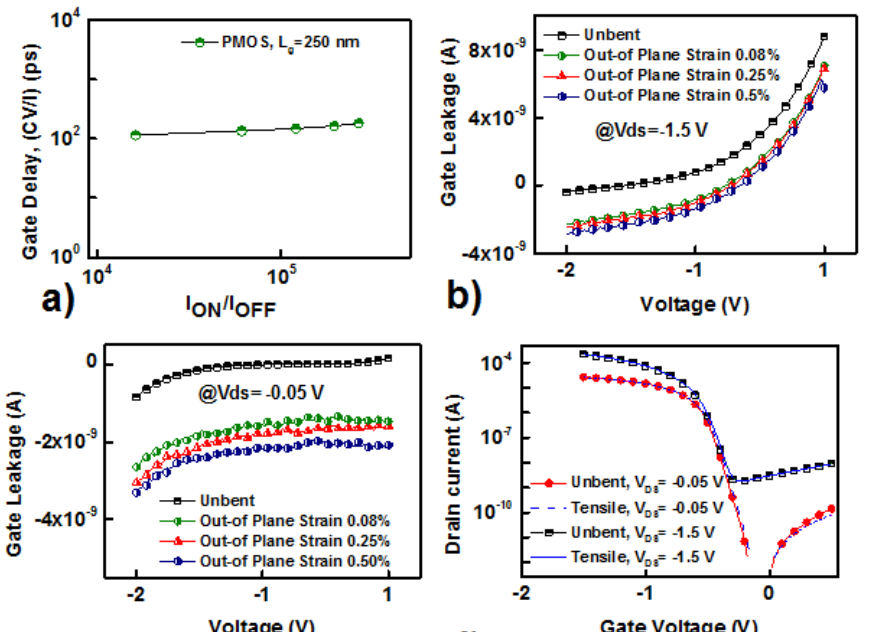

c)

b)

Voltage (V)

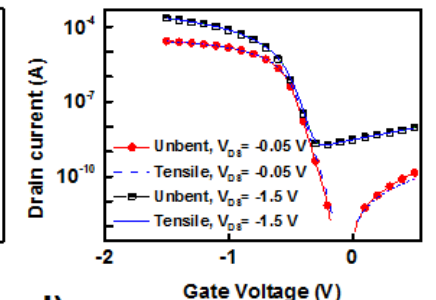

d)

Fig. 7. Gate delay (a) and leakage trends (b-c) for a $250 \mathrm{~nm}$ p-type FinFET with the transfer plot shown in (d).
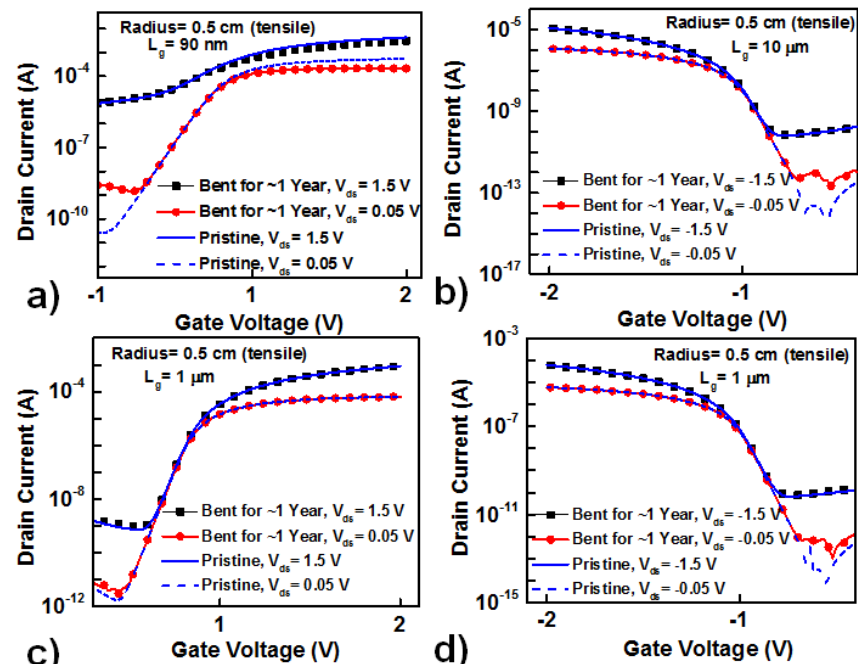

c)

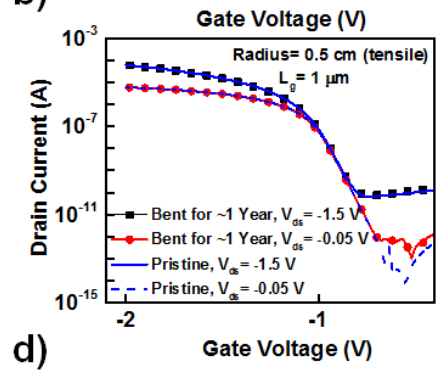

Fig. 8. Transfer plots for short and long channel FinFETs bent for 12 months at $0.5 \mathrm{~cm}$ bending radius (a-d).

This explains the minor deviations in the transfer and output $\mathrm{I}-\mathrm{V}$ curves concerning the effect on source-to-drain currents and gate leakage. Stress recovery and self-healing properties are widely used concepts in reliability physics, drawing the cutline between a stressed and post-stressed device. We have previously shown a self-healing property in metal-oxidesemiconductor capacitors (MOSCAPs) subjected to mechanical stresses [20]. The same study shows that for silicon based CMOS dynamic stress (successive bending and flattening) has severe effect on the reliability of the structure. Hence, they are more suitable for bent static structures than dynamic ones. Accelerated ramping voltage $\left(V_{\text {ramp }}\right)$ analysis, time dependent dielectric breakdown (TDDB), and bias temperature instability (BTI) tests used in assessing devices' useful projected lifetime employ various stress methods in which relaxation time has a strong effect on the measurements. For instance, one of the challenges in the reliability assessment of high- $\kappa$ dielectrics, including $\mathrm{HfSiO}_{x}$, is the accuracy of the BTI test composed of stress-measurestress cycles to track the shift in $V_{T H}$ due to the relaxation/recovery of degradation when stress is removed in the measurement step [21]. Fig. 9 shows the transfer I-V characteristics curves of long- and short-channel n- and p-type FinFET devices before and after applying stress. Evidently, the variation does not reflect the significant deviations experienced under effective stress as previously depicted in Fig. 3. The results of the flexible microcrystalline silicon TFT show that devices can be folded for storage then unfolded when needed, recovering their characteristics after being reflattened [19]. In their work, Dong et al. used 150-nm-thick silicon nitride $\left(\mathrm{Si}_{3} \mathrm{~N}_{4}\right)$ as a gate insulator. Although $\mathrm{Si}_{3} \mathrm{~N}_{4}$ was shown to exhibit high trap densities, which is expected to worsen with bending due to increased dielectric stress, it was shown that relaxation/recovery time decreased as the $\mathrm{Si}_{3} \mathrm{~N}_{4}$ thickness increases [22], thus manifesting the durability of the $\mathrm{Si}_{3} \mathrm{~N}_{4}$ TFTs when re-flattened. This work shows that this conclusion can be extended to scaled high-performance high- $\kappa$ dielectric FinFETs that use thin (4-nm-thick) HfSiO gate insulators.
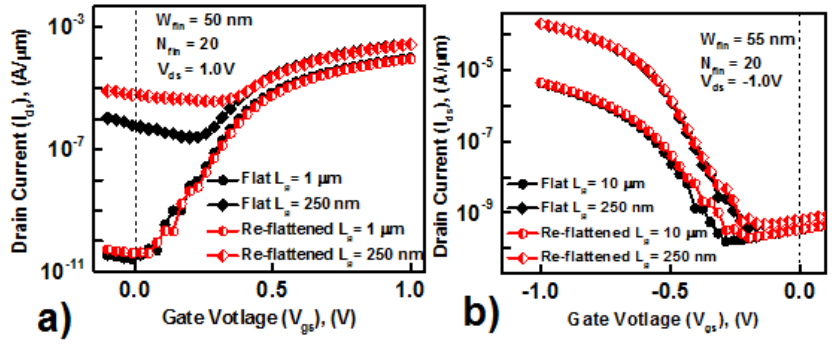

Fig. 9. Transfer plots for re-flattened n-type (a) and p-type (b) FinFETs.

\section{CONCLUSION}

We have presented a comprehensive analysis on varying flexed (bent) states of flexible high-performance FinFET CMOS. We have shown this reduces stress recovery when devices are re-flattened. Nonetheless, due to the sensitivity of the measurements, an extra source of variation (i.e. contact resistance) is added. As previously mentioned, the outlined percentage variations are ultimately useful for the actual systems applications, whether it is through flexible ICs for the internet of things (IoT), IoE, or wearable electronics. The results show that most variations are restricted to $\pm 10 \%$, which is similar to the process variations range in deep-submicron technologies where possible mitigation solutions are already practiced; however, compromised gate dielectrics introduce new reliability concerns for devices functioning in the bent state. Moreover, we provide insight into the degree of variation in device characteristics when they are bent, which complements previous work and shows that variations can be 
mitigated to render the devices suitable for performing while bent as well as when flattened. This work presents the possibility of adding the flexing ability to industry-grade

\section{REFERENCES}

[1] J. Zhang, M. De Marchi, P.-E. Gaillardon, and G. De Micheli, "A Schottky-Barrier Silicon FinFET with $6.0 \mathrm{mV} / \mathrm{dec}$ Subthreshold Slope over 5 Decades of Current," in Proc. IEEE Int. Electron Devices Meeting, 2014, No. EPFL-CONF-201905.

[2] G. Moore. (1965). Cramming More Components Onto Integrated Circuits, Electronics. Proc. IEEE [Online]. 86(1), pp. 82-85. Available: http://ieeexplore.ieee.org/xpls/abs_all.jsp?arnumber $=658762$

[3] E. Yu, L. Chang, S. Ahmed, H. Wang, S. Bell, C. Y. Yang, C. Tabery, C. Ho, Q. Xiang, T. J. King, and J. Bokor, , "FinFET scaling to $10 \mathrm{~nm}$ gate length," in Proc. IEEE Int. Electron Devices Meeting, 2002, pp. 251-254.

[4] The International Technology Roadmap for Semiconductors, "Table ORTC-1: ITRS Technology Trend Targets," Semicond. Ind. Assoc., Washington, DC, USA, 2013.

[5] T.-i. Kim, Y. H. Jung, H.-J Chung, K. J. Yu, N. Ahmed, C. J. Corcoran, J. S. Park, S. H. Jin, and J. A. Rogers. (2013). Deterministic assembly of releasable single crystal silicon-metal oxide field-effect devices formed from bulk wafers. Appl. Phys. Lett. [Online]. 102 (18), pp. 182104. Available: http://scitation.aip.org/content/aip/journal/apl/102/18/10.1063/1.480413 $\underline{9}$

[6] S. A. Stauth and B. A. Parviz. (2006). Self-assembled single-crystal silicon circuits on plastic. Proc. Natl. Acad. Sci. [Online]. 103(28), pp. 13922-13927. Available: http://www.pnas.org/content/103/38/13922.full

[7] G. A. T. Sevilla, J. P. Rojas, H. M. Fahad, A. M. Hussain, R. Ghanem, C. E. Smith, and M. M. Hussain. (2014, May). Flexible and Transparent Silicon-on-Polymer Based Sub-20 nm Non-planar 3D FinFET for BrainArchitecture Inspired Computation. Adv. Mater. [Online]. 26(18), pp. 2794-2799.

Available: http://onlinelibrary.wiley.com/doi/10.1002/adma.201305309/abstract

[8] J. Wu, C. Huang, and C. Liao, "Fracture strength characterization and failure analysis of silicon dies," Microelectron. Rel., vol. 43, no. 2, p. 269-277, Feb. 2003. [Online]. Available: http://www.sciencedirect.com/science/article/pii/S0026271402003141

[9] Z. Liu, Y. Huang, L. Xiao, P. Tang, and Z. Yin. (2015, Mar.). Nonlinear characteristics in fracture strength test of ultrathin silicon die. Semicond. Sci. Techol. [Online]. 30(4), pp. 045005. Available: http://iopscience.iop.org/article/10.1088/0268-

1242/30/4/045005/meta;jsessionid=F652D4CD866BA99D001F1C319E 0B7BAB.ip-10-40-2-108

[10] E. J. Boyd and D. Uttamchandani. (2012, Feb.). Measurement of the anisotropy of young's modulus in single-crystal silicon. $J$. Microelectromech. Syst. [Online]. 21(1), pp. 243-249. Avialable: http://ieeexplore.ieee.org/stamp/stamp.jsp?arnumber=6093930

[11] T. Sekitani, U. Zschieschang, H. Klauk, and T. Someya. (2010, Nov.). Flexible organic transistors and circuits with extreme bending stability. Nat. Mater.[Online]. 9, pp. 1015-1022. Available: http://www.nature.com/nmat/journal/v9/n12/abs/nmat2896.html

[12] J. Dolbow and M. Gosz. (1996, Aug.). Effect of out-of-plane properties of a polyimide film on the stress fields in microelectronic structures Mech. Mater. [Online]. 23(4), pp. 311-321. Available: http://www.sciencedirect.com/science/article/pii/016766369600021X

[13] S. E. Thompson, M. Armstrong, C. Auth, M. Alavi, M. Buehler, R. Chau, S. Cea, T. Ghani, G. Glass, T. Hoffman, and C. H. Jan. (2004, Nov.). A 90-nm logic technology featuring strained-silicon. IEEE Trans. Electron Devices [Online]. 51(11), pp. 1790-1797. Available: http://ieeexplore.ieee.org/xpls/abs_all.jsp?arnumber=1347396

[14] J. Welser, J. Hoyt, and J. Gibbons, "NMOS and PMOS transistors fabricated in strained silicon/relaxed silicon-germanium structures," in Proc. IEEE Int. Electron Devices Meeting, 1992, pp. 1000-1002.

[15] K. Rim, J. Chu, H. Chen, K. A. Jenkins, T. Kanarsky, K. Lee, A. Mocuta, H. Zhu, R. Roy, J. Newbury, and J. Ott,., "Characteristics and device design of sub-100 nm strained Si N-and PMOSFETs," in Symp. VLSI Technol., Dig. Tech. Pap., 2002, pp. 98-99.

[16] L. Dobrescu, M. Petrov, and C. Ravariu, "Threshold voltage extraction methods for MOS transistors," in Proc. Semicond. Conf., 2000, pp. 371374.
FinFETs without sacrificing functionality and highlights the various effects that out-of-plane bending has on these devices.

[17] Y. S. Choi, T. Numata, T. Nishida, R. Harris, and S. E. Thompson. (2008, Mar.). Impact of mechanical stress on gate tunneling currents of germanium and silicon p-type metal-oxide-semiconductor field-effect transistors and metal gate work function. J. Appl. Phys.[Online]. 103(6), pp. $064510 . \quad$ Available: http://scitation.aip.org/content/aip/journal/jap/103/6/10.1063/1.2838234

[18] K. Goto, A. Fushida, J. Watanabe, T. Sukegawa, K. Kawamura, T. Yamazaki, and T. Sugii, "Leakage mechanism and optimized conditioms of Co salicide process for deep-submicron CMOS devices," in Proc. IEEE Int. Electron Devices Meeting, 1995, pp. 449-452.

[19] H. Dong, Y. Kervran, N. Coulon, O. De Sagazan, E. Jacques, and T. Mohammed-Brahim. (2015, Oct.). Highly Flexible Microcrystalline Silicon n-Type TFT on PEN Bent to a Curvature Radius of $0.75 \mathrm{~mm}$. IEEE Trans. Electron Devices [Online]. 62(10), pp. 3278-3284. Available: http://ieeexplore.ieee.org/stamp/stamp.jsp?arnumber $=7239582$

[20] M. Ghoneim, A. Kutbee, F. G. Nasseri, G. Bersuker, and M. Hussain. (2014, Jun.). Mechanical anomaly impact on metal-oxide-semiconductor capacitors on flexible silicon fabric. Appl. Phys. Lett. [Online]. 104(23), 234104 Available: pp. http://scitation.aip.org/content/aip/journal/apl/104/23/10.1063/1.488264 7

[21] B. H. Lee, R. Choi, J. H. Sim, S. A. Krishnan, J. J. Peterson, G. A. Brown, and G. Bersuker.(2005, Mar.). Validity of constant voltage stress

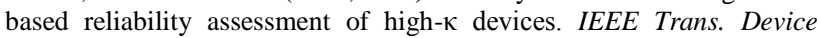
Mater. Rel. [Online]. 5(1), pp. 20-25. Available: http://ieeexplore.ieee.org/xpls/abs all.jsp?arnumber $=1435385$

[22] U. Zaghloul, G. Papaioannou, F. Coccetti, P. Pons, and R. Plana. (2009, Sep.). Dielectric charging in silicon nitride films for MEMS capacitive switches: Effect of film thickness and deposition conditions. Microelectron. Rel. [Online]. 49(9), pp. 1309-1314. Avaialable: http://www.sciencedirect.com/science/article/pii/S0026271409002340

Mohamed T. Ghoneim (S'13) is currently pursuing the Ph.D. degree. His current research interests include non-volatile memories, reliability testing, and heat dissipation of IoT electronics.

Nasir Alfaraj is currently pursuing the Ph.D. degree.

His current research interests include electrical characterization.

Galo A. Torres-Sevilla (S'13) is currently pursuing the Ph.D. degree. His current research interests include integration of high performance planar and non-planar CMOS on standard and flexible platform.

Hossain M. Fahad (S'09) received the Ph.D. degree.

$\mathrm{He}$ is currently holds a postdoctoral position with the University of California at Berkeley, Berkeley, CA, USA. His current research interests include CMOS integrated sensory system.

Muhammad M. Hussain (M'07-SM'10) is currently an Associate Professor and an IEEE Electron Devices Society Distinguished Lecturer. His current research interests include expansion of CMOS technology for futuristic applications. 